\title{
The Effects of Storage Conditions on the Stability of Porcine Placenta Extract-loaded Liposome Formulations
}

\author{
Sang Myoung Noh${ }^{1}$, Da-Eui Park ${ }^{2}$, Saewon $\mathrm{Im}^{1}$, Sunil Kim ${ }^{1}$, Young Bong Kim ${ }^{3}$ and Yu-Kyoung $\mathrm{Oh}^{1 \uparrow}$ \\ ${ }^{1}$ School of Pharmacy, Seoul National University, Seoul, South Korea \\ ${ }^{2}$ School of Life Science and Biotechnology, Korea University, Seoul, South Korea \\ ${ }^{3}$ Department of Animal Biotechnology, Konkuk University, Seoul, South Korea
}

(Received May 16, $2010 \cdot$ Revised June 1, $2010 \cdot$ Accepted June 1, 2010)

\begin{abstract}
We aimed to evaluate the effect of temperature, $\mathrm{pH}$, and light conditions on the stability of porcine placental extract (PPE)-loaded liposomes with different surface charges. The size distribution profiles and in vitro release patterns were investigated by dynamic light scattering method and spectrophotometry. The stability of PPE-loaded liposomes was affected by the surface charges of the liposomes. As compared to neutral and anionic liposomes, cationic liposome formulations showed significantly lower physical stability. At the test storage conditions of different temperatures and $\mathrm{pHs}$, the mean sizes of cationic PPE-loaded liposomes substantially increased. In contrast, neutral and anionic liposomes did not reveal significant changes in mean sizes upon various storage conditions. The neutral and anionic liposomes showed no significant differences in the release profiles of PPE after storage at various temperatures and pHs. Our results indicate that anionic and neutral liposome compositions might be more suitable for the formulations of PPE providing the higher stability.
\end{abstract}

Key words - Surface charges, Stability of liposome, Placenta extracts

Liposomal formulations have been studied for sustained delivery of small chemicals and peptide/protein drugs. (Kaasgaard et al., 2010; Marcato et al., 2008). To develop liposomes as carriers of bioactive substances, the stability at various temperature and $\mathrm{pH}$ must be considered. The stability of liposomes during storage mainly depends on the physic-chemical properties of the lipid membrane composition. (Eliyahu et al., 2007; Ntimenou et al., 2006; Sulkowski et al., 2005). Lipid-bilayer and membrane-surface characteristics, and particle sizes are the critical factors that affect the liposomal behavior and drug release dynamics. (Li et al., 2010). The stability of liposomes has been also reported to be affected by the storage conditions. (Pietzyk et al., 2000).

Emulsions and liposomes have been studied as topical formulations of porcine placenta extracts (PPE). (Kim et al., 2007). However, the effects of surface charges on the stability of the PPE-loaded liposome formulations have not been elucidated. In this study, we tested whether the surface charges of various PPE-loaded liposomes could affect the physical stability of the liposomes and in vitro release rate of PPE. The stability of liposomes was evaluated under storage at various temperature, $\mathrm{pH}$, and light conditions. Here, we report that the liposomal surface charges may govern the physical stability of

Corresponding Author:

Tel : +82-2-880-2493, E-mail : ohyk@snu.ac.kr

DOI : 10.4333/KPS.2010.40.3.187 liposomes during storage, indicating the importance of liposome composition for the placental extract formulation.

\section{Materials and Methods}

\section{Materials}

1- $\alpha$-Egg phosphatidylglycerol (PG), 1,2-dioleoyl-sn-glycero3-phosphoethanolamine (DOPE), 1,2-dioleoyl-3-trimethylammonium-propane (DOTAP), l- $\alpha$-egg phosphatidylcholine (PC) and cholesterol (Chol) were purchased from Avani Polar Lipids. Inc. (Birmingham, AL, USA). Porcine placenta extracts (PPE) was kindly provided from Dr. Jeong Ho Kim (Seogang University, Seoul, South Korea).

\section{Liposome Preparation}

To prepare liposomes of different surface charges, lipids were dissolved in chloroform with various compositions. The compositions of liposomes were PC/Chol (1/1, mole/mole) for neutral, DOTAP/DOPE (1/1, mole/mole) for cationic, and PG/ $\mathrm{PC} / \mathrm{Chol}$ (1/1/1, mole/mole) for anionic liposomes, respectively. The lipid mixtures were placed in a rotary evaporator to remove the chloroform and obtain thin lipid films. For the entrapment of PPE into the liposomes, lipid films were hydrated with $1 \mathrm{~mL}$ of PPE in phosphate buffered saline solution (PBS, $\mathrm{pH} 7.4$, containing PPE at a concentration of $5 \mathrm{mg}$ / $\mathrm{mL}$ ). After vortexing, the liposomes were extruded three times through $0.2 \mu \mathrm{m}$ polycarbonate membrane filter using an 
extruder (Northern Lipids, British Columbia, Canada). The PPE-loaded liposomes were passed through PD-10 column to remove the unentrapped PPE.

\section{Size Measurement of Liposomes}

The analysis of size distribution of PPE-loaded liposomes was determined by dynamic light scattering technique. The samples were diluted in PBS and/or 0.1 M sodium phosphate buffer and placed into a four sided cuvette in an ELS-8000 light scattering instrument (Photal, Osaka, Japan). The diameters of the liposomes were measured via He-Ne laser (10 $\mathrm{mW}$ ) light scattering at an angle of $90^{\circ}$ at $25^{\circ} \mathrm{C}$. An ELS-8000 software package supplied by the manufacturer was used to analyze the data.

\section{In vitro Release Test}

For in vitro release study, the PPE-loaded liposomes $(1 \mathrm{~mL})$ were added to a dialysis bag having a pore size of $2.4 \mathrm{~nm}$ and molecular weight cutoff between 12,000 and $14,000 \mathrm{kD}$. The dialysis tubing with PPE-loaded liposomes was placed in $5 \mathrm{~mL}$ of PBS and maintained at $25^{\circ} \mathrm{C}$. At various time intervals of $0.5,1,2,3,6,12$ and $24 \mathrm{hrs}, 200 \mu \mathrm{L}$ of the sample was collected. The amounts of PPE in each sample were measured by bicinchoninic acid assay (BCA) method. An aliquot $(150 \mu \mathrm{L})$ of the samples were added to $100 \mu \mathrm{L}$ of BCA solution. The samples were mixed while shaking at $300 \mathrm{rpm}$ at $37^{\circ} \mathrm{C}$ for 30 min. The optical density values were measured at $570 \mathrm{~nm}$ using a spectrophotometer Zenyth 3100 (Anthos Labtec., Salzburg, Austria). The calibration curves of protein concentrations were established using bovine serum albumin (BSA) solutions.

\section{Liposome Stability Test}

To examine the stability of liposomes by temperature, $\mathrm{pH}$, and light conditions, the PPE-loaded liposomes $(1 \mathrm{~mL})$ were divided into three aliquots and incubated at different conditions. To evaluate the thermal stability of liposomes, aliquots were incubated at 4 and $25^{\circ} \mathrm{C}$ for 7 days, respectively. For examination of $\mathrm{pH}$ stability, liposomes aliquots were stored at $0.1 \mathrm{M}$ sodium phosphate buffer with different $\mathrm{pH}$ values $(\mathrm{pH}$ $5,6,7$, and 8) at room temperature for 7 days. Furthermore, aliquots of liposomes were placed at with or without light conditions for 7 days to evaluate the light stability of liposomes at room temperature.

\section{Statistic Analysis}

Statistical analysis of data was performed using ANOVA, with the Student-Newman-Keuls test employed as a post-hoc test. SigmaStat software (version 3.5, Systat Software, Richmond, CA, USA) was used for all analyses. A $p$ value of less than 0.05 was considered significant.

\section{Results and Discussions}

Effect of Temperatures and Light on the Stability of

\section{PPE-loaded Liposomes}

The storage temperature of liposomes affected the stability of PPE-loaded liposomes in composition-dependent manner. The effect of storage temperatures on the stability of different surface charged PPE loaded-liposomes was examined. Anionic and neutral surface charges of PPE loaded-liposome did not show significant differences in size distribution patterns after 7-day storage at 4 (Fig. 1A) and $25^{\circ} \mathrm{C}$ (Fig. 1B). The mean size of anionic PPE-loaded liposomes was $162.5 \pm 15.7 \mathrm{~nm}$ on the preparation day. At 7 days after storage at $4^{\circ} \mathrm{C}$, the mean size of anionic liposomes was $142.3 \pm 5.9 \mathrm{~nm}$. The mean sizes of neutral liposomes ranged from 180 to $230 \mathrm{~nm}$ upon storage at 4 and $25^{\circ} \mathrm{C}$ for 7 days. In contrast, the mean sizes of cationic PPE-loaded liposomes increased 8.9- and 11.8-fold after storage at 4 and $25^{\circ} \mathrm{C}$ for 7 days, respectively. Similarly, 10.2-fold increase was observed in the mean size of cationic PPE-loaded liposomes after storage under dark condition at $25^{\circ} \mathrm{C}$ for 7 days (Fig. 1C).

The instability of cationic PPE-loaded liposomes during storage at light (Fig. 1B) and dark (Fig. 1C) conditions suggest that the instability of the liposomes might be not due to the light. Rather, it is more probable that the instability of the cationic PPE-loaded liposomes might be caused by the interaction of the components of PPE with cationic moieties of liposomes. In this study, all the liposomes were extruded using a polycarbonate filter of $200 \mathrm{~nm}$. Notably, the cationic PPE-loaded liposomes showed higher mean sizes than neutral and anionic liposomes from the preparation day. Given the higher mean size of cationic PPE liposomes on day 0, we can't exclude the possibility that the components of PPE interacting with cationic parts may be involved in the more than 10-fold of abrupt increase at at $25^{\circ} \mathrm{C}$ for 7 days. It is unlikely that empty cationic liposome per se is unstable as compared to the anionic and neutral liposomes. In case of liposomes containing other chemical drug, temoporin, cationic liposomes have been reported to show the higher stability than anionic liposomes. (DragicevicCuric et al., 2010). Moreover, we observed that empty cationic liposomes did not show significant increase in sizes even after 7 day storage (data not shown).

The liposomal formulations have been optimized for the properties of bioactive substances. In this study, we aimed to 

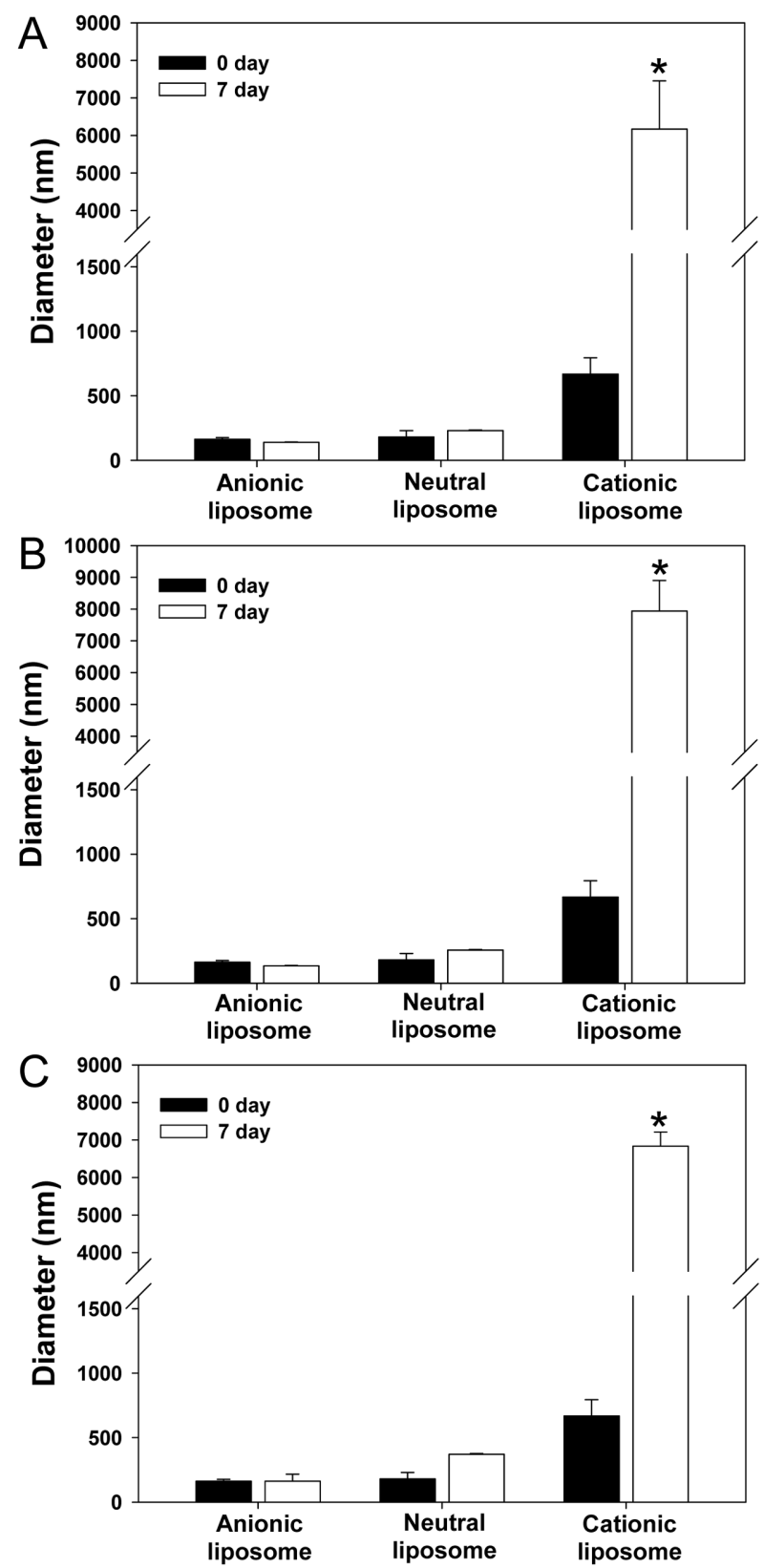

Figure 1. Effects of temperature and light on the mean sizes of PPE-loaded liposomes.

The mean sizes of various PPE-loaded liposomes were measured at day 0 and day 7 after storage at $4^{\circ} \mathrm{C}(\mathrm{A}), 25^{\circ} \mathrm{C}(\mathrm{B})$, and $25^{\circ} \mathrm{C}(\mathrm{C})$ under dark conditions. The $\mathrm{pH}$ of liposomal solution was fixed as 7.4. The results are expressed as the mean $\pm \mathrm{SD}(\mathrm{n}=3)$. *: significantly different from anionic and neutral liposomes (ANOVA and StudentNewman-Keuls test, $\mathrm{p}<0.05$ ).

figure out the optimal formulations for liposomes entrapping PPE. Due to the nature of complex components of PPE, we speculated that the charges of liposomal formulations may influence the stability of liposomes in different $\mathrm{pHs}$ and during storage periods. Indeed, we observed that cationic lipo- somes may not be suitable formulations for PPE in terms of physical stability due to the huge increase of sizes during storage.

Seven days are not sufficient time to test the stability of formulations. However, we think that this period can be used to screen out the most unstable formulations in relatively short periods. Indeed, we figured out that cationic liposomal formulations may have stability problems even after 7 day storage. Longer period of storage test should be done further in the near future.

\section{Effect of pH on the Stability of PPE-loaded Liposomes}

Although the anionic and neutral liposomes did not show significant differences in mean sizes upon storage at different temperature and light conditions, the anionic and neutral liposomes showed the increases in the mean sizes at acidic $\mathrm{pH}$ conditions. At $\mathrm{pH} 5$, the mean sizes of anionic liposomes increased from $236 \pm 14.8 \mathrm{~nm}$ at day 0 to $696 \pm 131.4 \mathrm{~nm}$ after storage at $\mathrm{pH} 5$ for 7 days (Fig. 2A). In neutral liposomes, the mean size was the highest after storage at $\mathrm{pH} 5$ for 7 days (Fig. 2B). However, at other $\mathrm{pH}$ conditions the increase of the liposomal mean sizes was not significant or minimal for both anionic (Fig. 2A) and neutral (Fig. 2B) liposomes.

Meanwhile, the instability of cationic PPE-loaded liposomes was consistently observed at all the $\mathrm{pH}$ conditions tested in this study (Fig. 2C). The mean sizes of cationic PPE-loaded liposomes were the highest after storage at $\mathrm{pH} 7.0$ for 7 days, showing 9.6-fold increase. The lowest change in the mean sizes of cationic liposomes was observed after storage at $\mathrm{pH}$ 6.0 .

\section{In vitro Release Profile of PPE from Liposomes}

Since the cationic PPE-loaded liposomes showed substantial instability during storage, we tested the in vitro release profile of PPE only from neutral and anionic liposomes stored at different temperatures. To test whether anionic and neutral liposomes show different patterns of PPE release regardless of stability, we first measured the in vitro profile of PPE from those two types of freshly prepared liposomes. Fig. 3A shows that there was no significant difference in the release pattern of PPE from neutral and anionic liposomes at day 0 . After storage for 7 days at $4^{\circ} \mathrm{C}$, anionic liposomes revealed similar patterns of PPE release to neutral liposomes (Fig. 3B). Even after the storage at $25^{\circ} \mathrm{C}$, anionic and neutral liposomes exhibited the similar rates of PPE release (Fig. 3C).

The $\mathrm{pH}$ of storage conditions affected the release of PPE from anionic liposomes. When anionic PPE-loaded liposomes were stored at $\mathrm{pH}$ values of 6 or 7 , the release patterns of PPE 

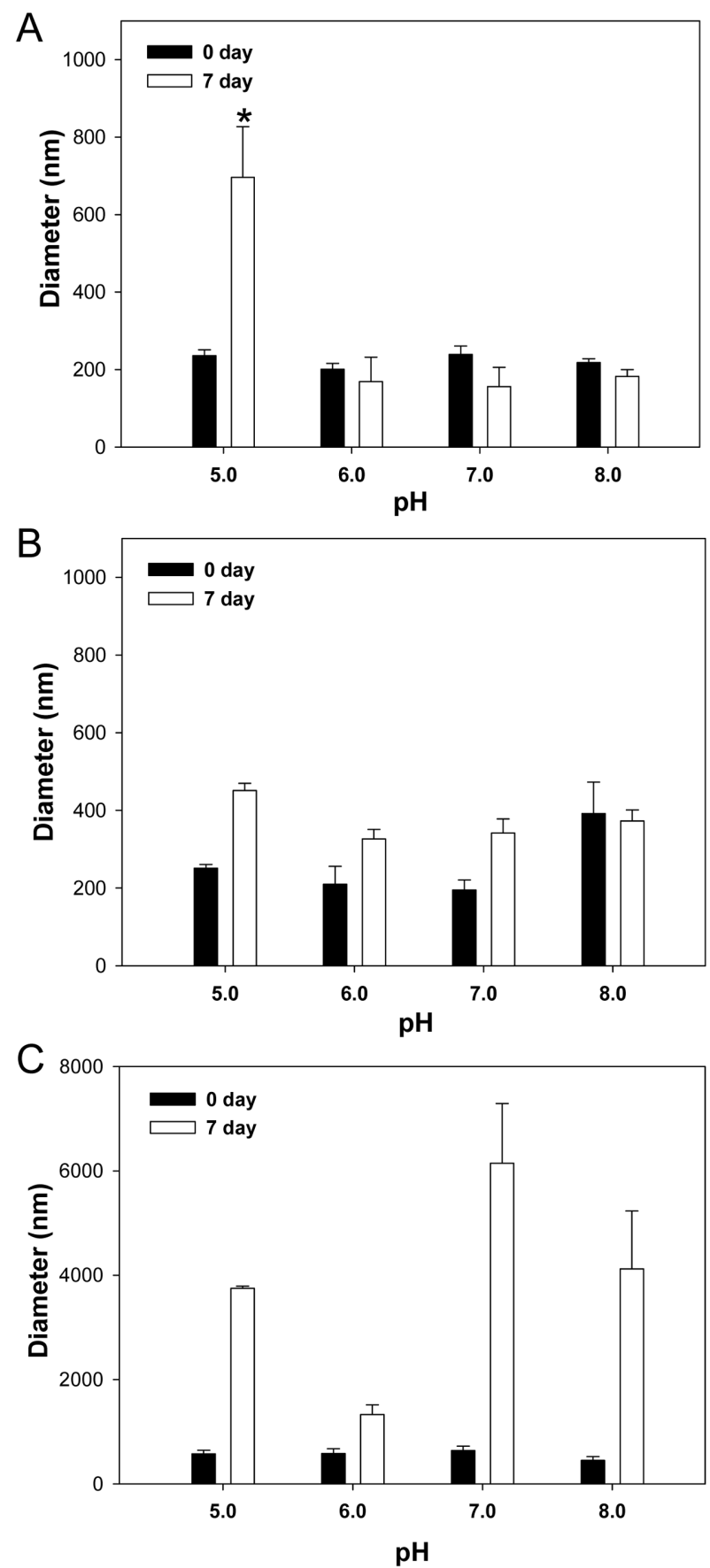

Figure 2. Effect of $\mathrm{pH}$ on the mean sizes of PPE-loaded liposomes. The mean sizes of PPE-loaded anionic (A), neutral (B), and cationic (C) liposomes were measured at day 0 and day 7 after storage at various $\mathrm{pH}$ conditions. All the liposomes were stored at $25^{\circ} \mathrm{C}$. The results are expressed as the mean $\pm \mathrm{SD}(\mathrm{n}=3) . *$ : significantly different other $\mathrm{pH}$ conditions (ANOVA and Student-Newman-Keuls test, $\mathrm{p}<0.05$ ).

did not differ. The amounts of PPE from anionic liposomes were higher after storage at $\mathrm{pH} 8$ than at other $\mathrm{pH}$ values (Fig. 4A). Similar to anionic liposomes, neutral liposomes showed
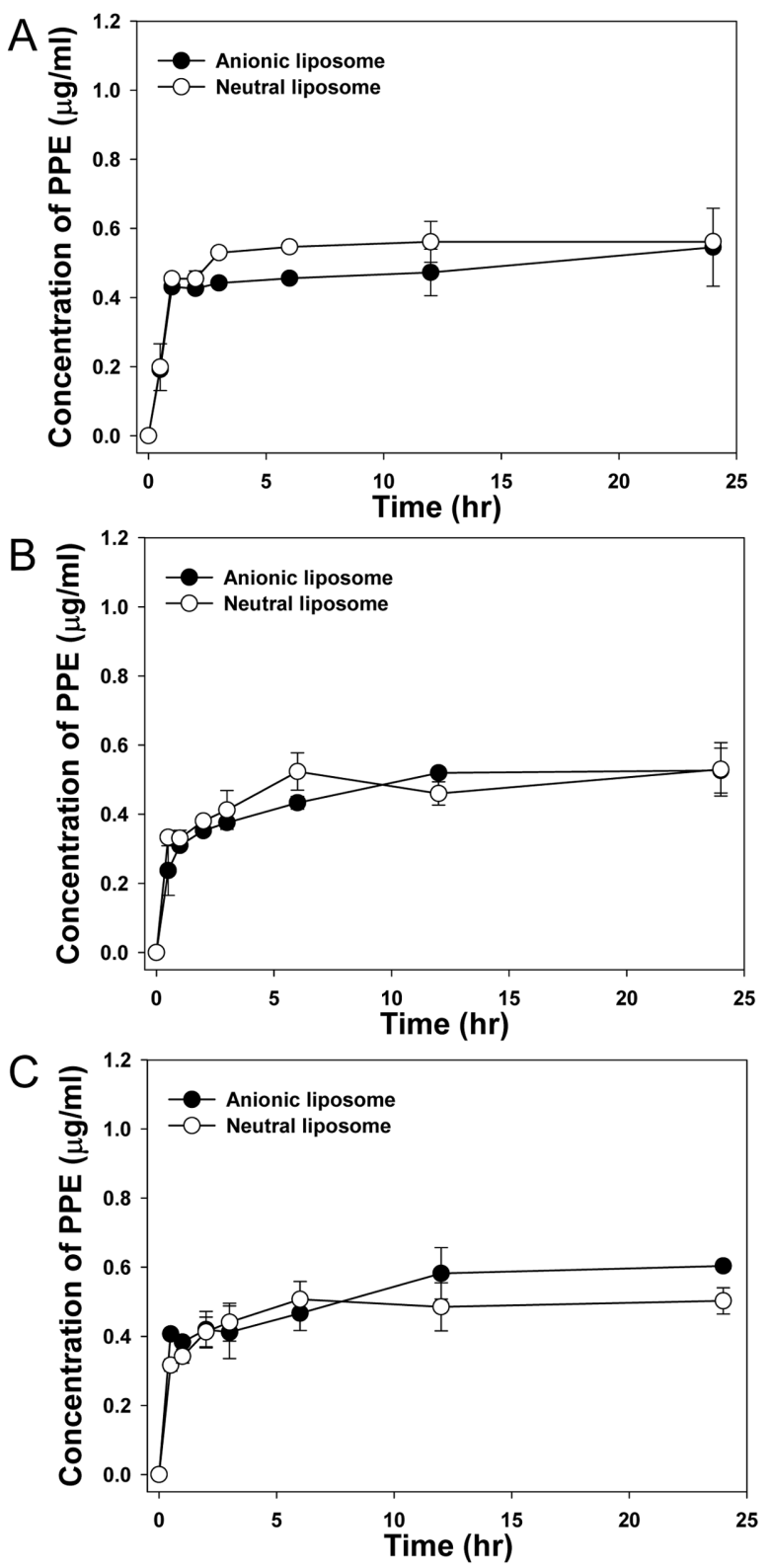

Figure 3. In vitro release of PPE from liposomes stored at various temperature and light conditions.

The in vitro release of PPE from liposomes was measured at day 0 (A) and 7 after storage at $4^{\circ} \mathrm{C}(\mathrm{B})$ and $25^{\circ} \mathrm{C}(\mathrm{C})$. The $\mathrm{pH}$ of liposomal solution was fixed as 7.4. The results are expressed as the mean \pm SD $(n=3)$.

the highest release of PPE after storage at $\mathrm{pH} 8$ (Fig. 4B). Given that the major components of PPE extracts are considered to be peptides and amino acids, the charges of major PPE components may have affected the stability and release behaviors from liposomal formulations.

Although the mean sizes of neutral and anionic liposomes did not significantly change (Fig. 2), we observed the highest increase of PPE release after storage at $\mathrm{pH} 8$ (Fig. 4). The dis- 

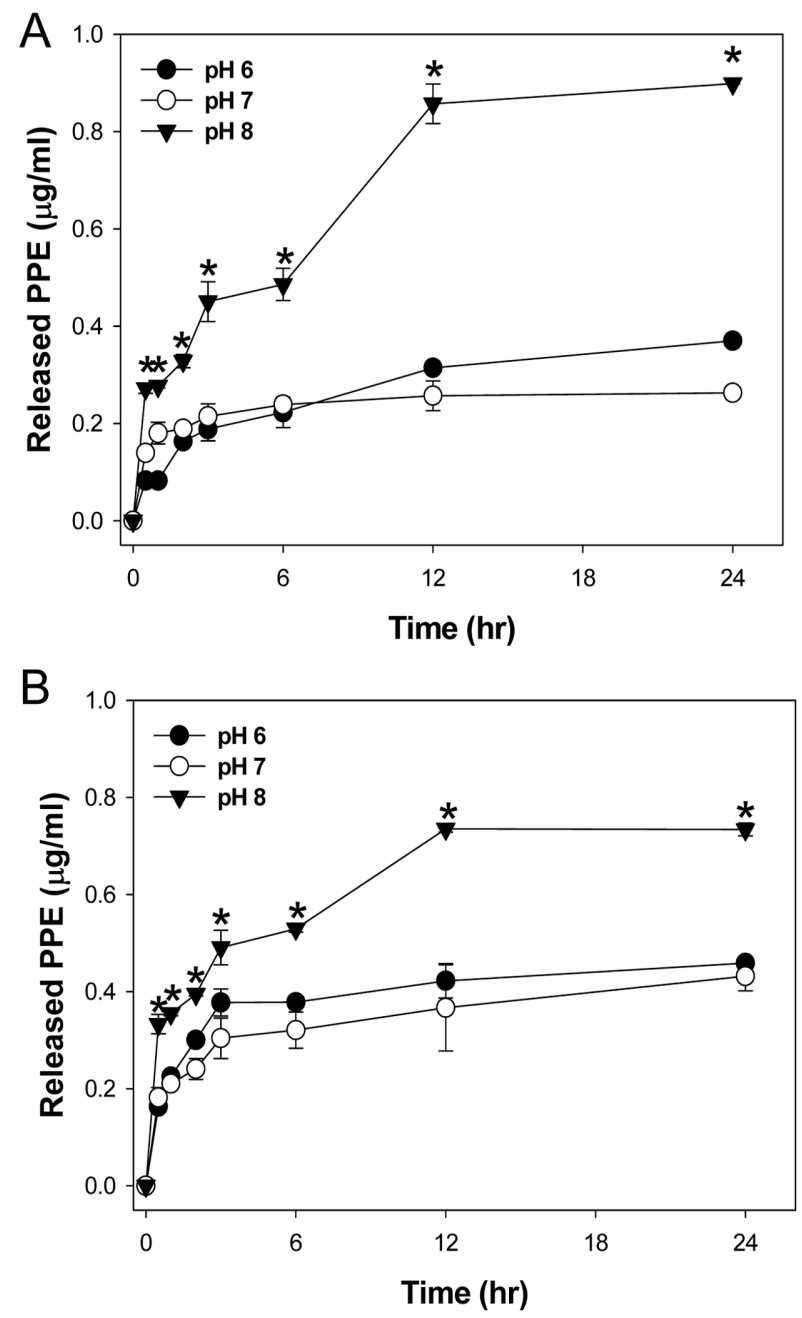

Figure 4. In vitro release of PPE from liposomes stored at various $\mathrm{pH}$ conditions.

The in vitro release of PPE from anionic (A) and neutral (B) liposomes was measured at day 7 after storage at various $\mathrm{pH}$ conditions. All the liposomes were stored at $25^{\circ} \mathrm{C}$. The results are expressed as the mean $\pm \mathrm{SD}(\mathrm{n}=3)$. * : significantly different other $\mathrm{pH}$ conditions (ANOVA and Student-Newman-Keuls test, $\mathrm{p}<0.05$ ).

crepancy between the similar mean sizes and different release pattern might be partly due to the chemical instability of PPEloaded liposomal membranes stored at $\mathrm{pH} 8$.

Our study on the stability of PPE-loaded liposomes at various $\mathrm{pH}$ conditions might be applied to the design of topical gels containing PPE-loaded liposomes. Previously, chlorophylline-loaded liposomes or temoporin-loaded liposomes have been formulated in carbomer-based hydrophilic gels for topical delivery. (Dragicevic-Curic et al., 2009; DragicevicCuric et al., 2005). Our observation on the physical stability of liposomes implies that $\mathrm{pH}$ ranges of 6-7 would be proper for the gel base of neutral or anionic PPE-loaded liposomes.

\section{Conclusion}

The physical stability of PPE-loaded liposomes was significantly affected by the surface charges of the liposomes. Unlike anionic and neutral liposomes, cationic PPE-loaded liposomes formed large aggregates upon storage at various temperatures. The size distribution and in vitro release study revealed that neutral liposomes composed of $\mathrm{PC} / \mathrm{Chol}$ and negatively charged PG/PC/Chol-based liposomes might be suitable formulations for prolonged storage of PPE. Moreover, this study indicates that the surface charges can play an important role for determining the physical stability of PPE-loaded liposomes.

\section{Acknowledgements}

This study was financially supported by the research grant from Rural Development Administration BioGreen 21 project (Code No. 20100301-061-200-001-03-00).

\section{References}

Dragicevic-Curic, N., Gräfe, S., Gitter, B., Winter, S., Fahr, A., 2010. Surface charged temoporfin-loaded flexible vesicles: in vitro skin penetration studies and stability. Int. J. Pharm. 384, 100-108.

Dragicevic-Curic, N., Winter, S., Stupar, M., Milic, J., Krajisnik, D., Gitter, B., Fahr A., 2009. Temoporfin-loaded liposomal gels: viscoelastic properties and in vitro skin penetration. Int. J. Pharm. 373, 77-84.

Dragicevic-Curic, N., Stupar, M., Miliæ, J., Zoriæ, T., Krajisnik, D., Vasiljeviæ, D., 2005. Hydrophilic gels containing chlorophyllin-loaded liposomes: development and stability evaluation. Pharmazie 60, 588-592.

Eliyahu, H., Joseph, A., Schillemans, J.P., Azzam, T., Domb, A.J., Barenholz Y., 2007. Characterization and in vivo performance of dextran-spermine polyplexes and DOTAP/cholesterol lipoplexes administered locally and systemically. Biomaterials 28 , 2339-2349.

Kaasgaard, T., Andresen, T.L., 2010. Liposomal cancer therapy: exploiting tumor characteristics. Expert Opin. Drug Deliv. 7, 225-243.

Kim, D.C., Noh, S.M., Park, K.T., Kim, Y.B., Baek, K.H., Oh, Y.K., 2007. Transdermal delivery of porcine placenta extracts using linolenic acid-based emulsion formulations. J. Kor. Pharm. Sci. 37, 281-286.

Li, S., Goins, B., Phillips, W.T., Bao, A., 2010. Remote-loading labeling of liposomes with 99mTc-BMEDA and its stability evaluation: effects of lipid formulation and $\mathrm{pH} /$ chemical gradient. J. Liposome Res. ePub. 
Marcato, P.D., Durán, N., 2008. New aspects of nanopharmaceutical delivery systems. J. Nanosci. Nanotechnol. 8, 22162229.

Ntimenou, V., Mourtas, S., Christodoulakis, E.V., Tsilimbaris, M., Antimisiaris, S.G., 2006. Stability of protein-encapsulating DRV liposomes after freeze-drying: A study with BSA and tPA. J. Liposome Res. 16, 403-416.
Pietzyk, B., Henschke, K., 2000. Degradation of phosphatidylcholine in liposomes containing carboplatin in dependence on composition and storage conditions. Int. J. Pharm. 196, 215218.

Sułkowski, W.W., Pentaka, D., Korus, W., Sułkowska, A., 2005. Effect of temperature on liposome structures studied using EPR spectroscopy. Spectroscopy 19, 37-42. 